\title{
PARAMETERIZATION AND MEASUREMENTS OF HELICAL MAGNETIC FIELDS
}

\author{
W. Fischer* and M. Okamura \\ Brookhaven National Laboratory, Upton, NY 11973, USA
}

\section{Abstract}

Magnetic fields with helical symmetry can be parameterized using multipole coefficients $\left(\tilde{a}_{n}, \tilde{b}_{n}\right)$. We present a parameterization that gives the familiar multipole coefficients $\left(a_{n}, b_{n}\right)$ for straight magnets when the helical wavelength tends to infinity. To measure helical fields all methods used for straight magnets can be employed. We show how to convert the results of those measurements to obtain the desired helical multipole coefficients $\left(\tilde{a}_{n}, \tilde{b}_{n}\right)$.

\section{INTRODUCTION}

The magnetic field inside straight magnets can be parameterized in terms of multipole coefficients $\left(a_{n}, b_{n}\right)$. We will present such a parameterization first. Fields of helical magnets (see Fig. 1) can be described in a similar way, by means of multipole coefficients $\left(\tilde{a}_{n}, \tilde{b}_{n}\right)$. We give a notation for the $\left(\tilde{a}_{n}, \tilde{b}_{n}\right)$ for which the $\left(a_{n}, b_{n}\right)$ are the limiting case when the helical wave length tends to infinity. A Cylindrical coordinate system $(r, \theta, s)$ is used where $s$ denotes the longitudinal direction.

We then assume that a magnetic field measurement device always parameterizes its measurements in terms of $\left(a_{n}, b_{n}\right)$, and give formulae to obtain the coefficients $\left(\tilde{a}_{n}, \tilde{b}_{n}\right)$ when a helical magnetic field is measured. Three types of measurement devices are treated: rotating "radial" coils, rotating "tangential" coils and rotating Hall probes (see Fig. 2 (a), (b) and (c) respectively).

\section{MAGNETIC FIELD PARAMETERIZATION}

\subsection{Straight Magnetic Fields}

In a current free region in vacuum where the electrical field $\vec{E}$ is constant, the magnetic field $\vec{B}$ can be derived from a scalar potential $\psi$ as $\vec{B}=-\nabla \psi$. We consider a magnet of infinite length, thus neglecting fringe fields. The symmetry condition of such an element is $\psi(r, \theta, s)=\psi(r, \theta, s+\Delta s)$ with $\Delta s$ arbitrary. Therefore, the potential $\psi$ is independent of $s, \psi(r, \theta, s)=\psi(r, \theta)$.

Having a main field $B_{0} \sin \theta$ the solution of the Laplace equation $\Delta \psi=0$ can be written as

$$
\begin{aligned}
\psi(r, \theta)= & -B_{0} \sum_{n=0}^{\infty} \frac{1}{n+1} \frac{r^{n+1}}{r_{0}^{n}} \times \\
& \times\left[a_{n} \cos ((n+1) \theta)+b_{n} \sin ((n+1) \theta)\right] .
\end{aligned}
$$

The $b_{n}$ are called "normal" and the $a_{n}$ "skew" multipole coefficients. Here, the subscript " 0 " denotes a dipole, " 1 " a

\footnotetext{
- Work performed under the auspices of the US Department of Energy
}

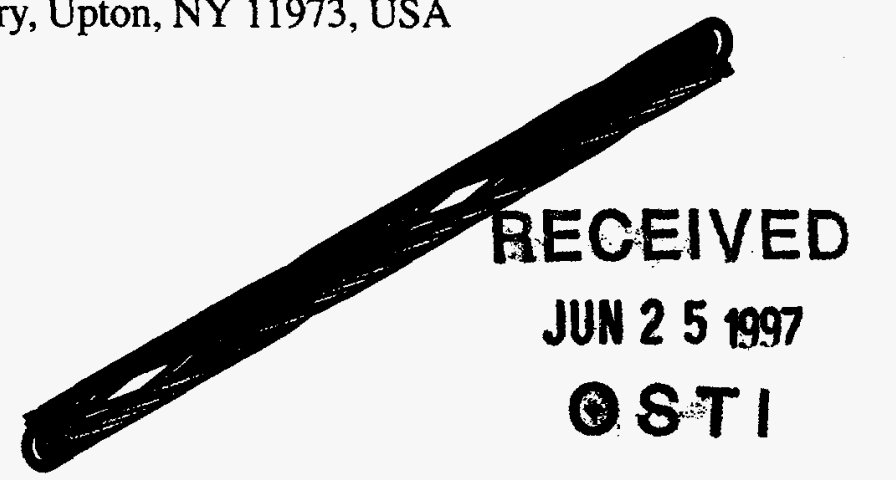

Figure 1: The conductor of a helical dipole (one helical wavelength long). This magnet will be used for proton spin manipulation in RHIC.

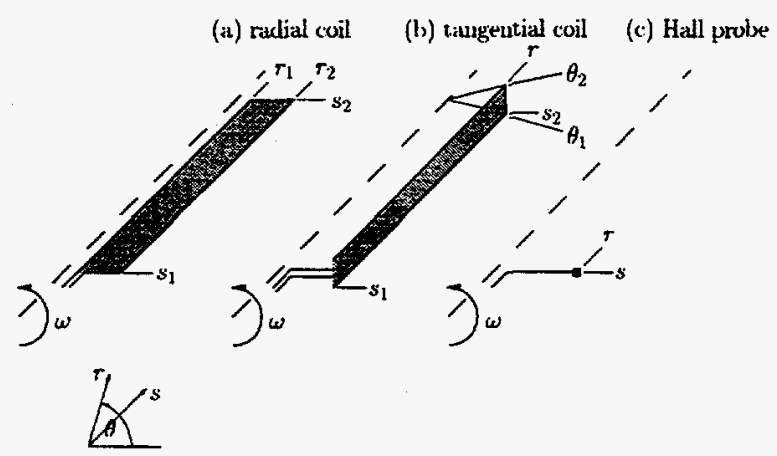

Figure 2: Three methods to measure magnetic multipole coefficients.

quadrupole etc. $r_{0}$ is a reference radius. ${ }^{1}$

From $\vec{B}=-\nabla \psi$ and Eq. (1) the magnetic field can be obtained. We have

$$
\begin{aligned}
& B_{r}=B_{0} \sum_{n=0}^{\infty}\left(\frac{r}{r_{0}}\right)^{n}\left[a_{n} \cos ((n+1) \theta)+b_{n} \sin ((n+1) \theta)\right], \\
& B_{\theta}=B_{0} \sum_{n=0}^{\infty}\left(\frac{r}{r_{0}}\right)^{n}\left[b_{n} \cos ((n+1) \theta)-a_{n} \sin ((n+1) \theta)\right], \\
& B_{s}=0 .
\end{aligned}
$$

\subsection{Helical Magnetic Fields}

We consider again a magnet of infinite length and neglect fringe fields. The symmetry condition for a helical magnet

\footnotetext{
${ }^{1}$ Note that the European notation (see for example Ref. [1]) differs from the American one presented here. The transformation is $b_{n, \text { American }}=b_{n+1, \text { European }}$ and $a_{n, \text { American }}=$ $-a_{n+1, \text { European. }}$.
} 
is $\psi(r, \theta, s)=\psi(r, \theta-k \Delta s, s+\Delta s)$, where $\Delta s$ is arbitrary. In other words, $\theta-k s=$ const. $k=2 \pi / \lambda$ is the wave number and $\lambda$ the wave length of the helix. $k$ shall have a positive sign for right-handed and a negative sign for lefthanded helices. Introducing the new variable $\tilde{\theta}=\theta-k s$, the symmetry condition leads to a potential $\psi$ which is only dependent on $r$ and $\tilde{\theta}, \psi(r, \theta, z)=\psi(r, \tilde{\theta})$. The tilde shall remind the reader of the fact that $\tilde{\theta}$ in a helix is similar to $\theta$ in a ordinary dipole. Using $(r, \tilde{\theta})$ as coordinates and having a transverse helical main Field $B_{0} \sin \tilde{\theta}$ a solution of the Laplace equation $\Delta \psi=0$ is

$$
\begin{aligned}
\psi(r, \tilde{\theta})= & -B_{0} \sum_{n=0}^{\infty} \frac{f_{n}}{k(n+1)} I_{n+1}((n+1) k r) \times \\
& \times\left[\tilde{a}_{n} \cos ((n+1) \tilde{\theta})+\tilde{b}_{n} \sin ((n+1) \tilde{\theta})\right]
\end{aligned}
$$

where $I_{n}$ are modified Bessel functions and the $f_{n}$ are defined as

$$
f_{n}=\frac{2^{n+1}(n+1) !}{(n+1)^{n+1}} \frac{1}{r_{0}^{n} k^{n}} .
$$

The $\tilde{b}_{n}$ are called "normal" and the $\tilde{a}_{n}$ "skew" helical multipole coefficients (with respect to the main field $B_{0}$ ). The subscript " 0 " denotes a helical dipole, the subscript " 1 " a helical quadrupole etc. $r_{0}$ is again a reference radius.

The factors in (5) are chosen in such a way as to obtain the potential (1) when the helical wave length tends to infinity. In this case $k \rightarrow 0, \tilde{\theta} \rightarrow \theta$ and the Bessel function can be approximated by $I_{n}(z) \approx \frac{1}{2^{n}} \frac{z^{n}}{n !}$ (cf. Ref. [2]). Now, the magnetic field can be computed as

$$
\begin{aligned}
B_{r}= & B_{0} \sum_{n=0}^{\infty} f_{n} I_{n+1}^{\prime}((n+1) k r) \times \\
& \times\left[\tilde{a}_{n} \cos ((n+1) \tilde{\theta})+\tilde{b}_{n} \sin ((n+1) \tilde{\theta})\right], \\
B_{\theta}= & -\frac{1}{k r} B_{s} \\
B_{s}= & -B_{0} \sum_{n=0}^{\infty} f_{n} I_{n+1}((n+1) k r) \times \\
& \times\left[\tilde{b}_{n} \cos ((n+1) \tilde{\theta})-\tilde{a}_{n} \sin ((n+1) \tilde{\theta})\right] .
\end{aligned}
$$

where $I_{n}^{\prime}$ denotes the derivative with respect to the argument of the Bessel function.

Since the Bessel function is nonlinear, a magnetic field with helical symmetry is nonlinear too, even the field of a perfect helical dipole. In addition, there is a longitudinal field component off the $s$-axis. Fig. 3 shows an example for the field inside a helical dipole.

\section{MEASUREMENTS OF HELICAL FIELDS}

While the multipole measurements with rotating coils are obtained from the field within the coil area (shaded in Fig. 2), the multipole coefficients of Hall probe measurements are obtained from the field on the circumference of a circle with given radius $r$. For rotating coils the magnetic flux is computed for both parameterizations $\left(a_{n}, b_{n}\right)$

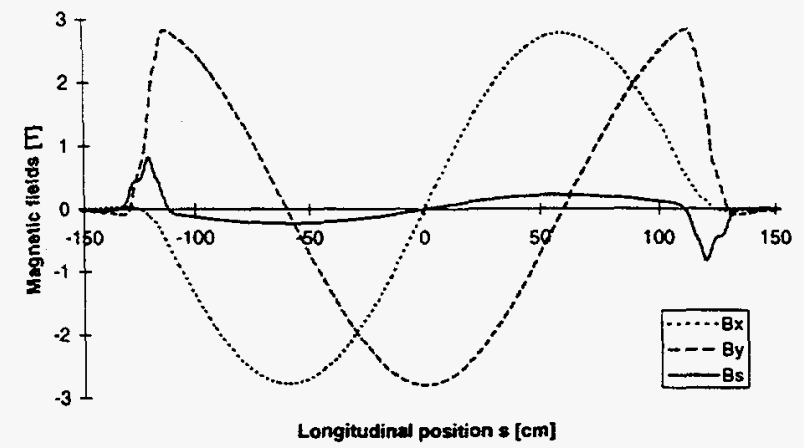

Figure 3: Magnetic field components along the $s$-axis with $x=0 \mathrm{~cm}$ and $y=31 \mathrm{~cm}$ for the magnet shown in Fig. 1. Note that end effects are not treated in this article.

and $\left(\tilde{a}_{n}, \tilde{b}_{n}\right)$. The results are stated in a form that allows a direct comparison. For Hall probe measurements the magnetic fields in both parameterizations are also stated in a form that allows a comparison. For all cases conversion formulae from $\left(a_{n}, b_{n}\right)$ to $\left(\tilde{a}_{n}, \tilde{b}_{n}\right)$ are given in the same form.

\subsection{Rotating Coils}

The magnetic flux through a coil is

$$
\Phi(\theta)=N \int \vec{B}(r, \theta) \cdot d \vec{a}
$$

where $\mathbf{N}$ is the number of coils windings. For rotating coils one has $\theta=\omega t$ and the induced voltage $U=-d \Phi / d t$ is proportional to the angular velocity $\omega$.

\subsubsection{Radial Coils}

The area of a flat rotating radial coil ranges from $r_{1}$ to $r_{2}$ and from $s_{1}$ to $s_{2}$ (cf. Fig. 2). The magnetic flux (10) through the coils is

$$
\Phi(\theta)=N \int_{s_{1}}^{s_{2}} \int_{r_{1}}^{r_{2}} B_{\theta}(r, \theta) d r d s .
$$

Using (3) the magnetic flux (11) for straight magnets becomes

$$
\left.\begin{array}{l}
\Phi(\theta)=N B_{0}\left(s_{2}-s_{1}\right) \sum_{n=0}^{\infty} K_{n}\left[b_{n} \cos ((n+1) \theta)-a_{n} \sin ((n+1) \theta)\right] \\
\text { with } K_{n}=\frac{r_{0}}{n+1}\left[\left(\frac{r_{2}}{r_{0}}\right)^{(n+1)}-\left(\frac{r_{1}}{r_{0}}\right)^{(n+1)}\right] .
\end{array}\right\}
$$

With (8) the flux for helical fields is

$$
\left.\begin{array}{l}
\Phi(\theta)=N B_{0}\left(s_{2}-s_{1}\right) \sum_{n=0}^{\infty} R_{n}\left[\hat{b}_{n} \cos ((n+1) \theta)-\hat{a}_{n} \sin ((n+1) \theta)\right] \\
\text { with } R_{n}=f_{n} \int_{r_{1}}^{r_{2}} \frac{1}{k r} I_{n+1}((n+1) k r) d r .
\end{array}\right\}
$$




\section{DISCLAMMER}

Portions of this document may be illegible in electronic image products. Images are produced from the best available original docoment. 

In (13) new magnetic multipole coefficients

$$
\begin{aligned}
& \hat{a}_{n}=+\tilde{a}_{n} T_{n}+\tilde{b}_{n} S_{n}, \\
& \hat{b}_{n}=-\tilde{a}_{n} S_{n}+\tilde{b}_{n} T_{n} .
\end{aligned}
$$

are used for which

$S_{n}\left(s_{1}, s_{2}\right)=-\frac{2}{\Delta s(n+1) k} \sin \frac{(n+1) k \Delta s}{2} \sin \frac{(n+1) k \Delta s}{2(15)}$

and

$T_{n}\left(s_{1}, s_{2}\right)=+\frac{2}{\Delta s(n+1) k} \sin \frac{(n+1) k \Delta s}{2} \cos \frac{(n+1) k \Delta s}{2(16)}$

with $\Delta s=s_{2}-s_{1}$ have been defined.

\subsubsection{Tangential Coils}

The magnetic flux through this type of coil is (cf. Fig. 2)

$$
\Phi(\theta)=N \int_{s_{1}}^{s_{2}} \int_{\theta_{1}}^{\theta_{2}} B_{r}(r, \bar{\theta}) r d \bar{\theta} d s .
$$

The difference $\Delta \theta=\theta_{2}-\theta_{1}$ is fixed and one can assume that $\theta_{1}=\theta-\Delta \theta / 2$ and $\theta_{2}=\theta+\Delta \theta / 2$ hold. With (2) the magnetic flux in straight magnets becomes

$$
\begin{aligned}
\Phi(\theta)= & N B_{0}\left(s_{2}-s_{1}\right) \cdot \frac{2}{n+1} \sin \frac{(n+1) \Delta \theta}{2} \times \\
& \times \sum_{n=0}^{\infty} K_{n}\left[a_{n} \cos ((n+1) \theta)+b_{n} \sin ((n+1) \theta)\right]
\end{aligned}
$$

and for helical fields one obtains with (7)

$$
\begin{aligned}
\Phi(\theta)= & N B_{0}\left(s_{2}-s_{1}\right) \cdot \frac{2}{n+1} \sin \frac{(n+1) \Delta \theta}{2} \times \\
& \times \sum_{n=0}^{\infty} R_{n}\left[\hat{a}_{n} \cos ((n+1) \theta)+\hat{b}_{n} \sin ((n+1) \theta)\right]
\end{aligned}
$$

The $\left(\hat{a}_{n}, \hat{b}_{n}\right)$ are defined by $(14)$ and the $\left(S_{n}, T_{n}\right)$ needed in this definition in $(15,16)$.

\subsection{Hall Probes}

For Hall probe measurements the magnetic fields, either tangential or radial, can be compared directly.

\subsubsection{Tangential Field Components}

The tangential field in a straight magnet is (cf. Eq. (3))

$$
\left.\begin{array}{l}
B_{\theta}(\theta)=B_{0} \sum_{n=0}^{\infty} K_{n}\left[b_{n} \cos ((n+1) \theta)-a_{n} \sin ((n+1) \theta)\right] \\
\text { with } K_{n}=\left(\frac{r}{r_{0}}\right)^{n}
\end{array}\right\}
$$

and in a helical field (cf. Eq. (8))

$$
\left.\begin{array}{l}
B_{\theta}(\theta)=B_{0} \sum_{n=0}^{\infty} R_{n}\left[\hat{b}_{n} \cos ((n+1) \theta)-\hat{a}_{n} \sin ((n+1) \theta)\right] \\
\text { with } R_{n}=\frac{1}{k r} f_{n} I_{n+1}((n+1) k r) .
\end{array}\right\}
$$

The $\left(\hat{a}_{n}, \hat{b}_{n}\right)$ are again defined by equations (14) and the $\left(S_{n}, T_{n}\right)$ needed in this definition by equations $(15,16)$.

\subsubsection{Radial Field Components}

The radial field in a straight magnet is cf. Eq. (2))

$$
\left.\begin{array}{l}
B_{r}(\theta)=B_{0} \sum_{n=0}^{\infty} K_{n}\left[a_{n} \cos ((n+1) \theta)+b_{n} \sin ((n+1) \theta)\right] \\
\text { with } K_{n}=\left(\frac{r}{r_{0}}\right)^{n}
\end{array}\right\}
$$

and in a helical field cf. Eq. (7))

$$
\left.\begin{array}{l}
B_{r}(\theta)=B_{0} \sum_{n=0}^{\infty} R_{n}\left[\hat{a}_{n} \cos ((n+1) \theta)+\hat{b}_{n} \sin ((n+1) \theta)\right] \\
\text { with } R_{n}=f_{n} I_{n+1}^{\prime}((n+1) k r) .
\end{array}\right\}
$$

Also in this case the $\left(\hat{a}_{n}, \hat{b}_{n}\right)$ are defined by equations (14) and the $\left(S_{n}, T_{n}\right)$ by equations $(15,16)$.

\subsection{Conversion}

It is now assumed that a device parameterizes the measured magnetic field in terms of multipole coefficients $\left(a_{n}, b_{n}\right)$ for straight magnets. If the measured magnetic field has helical symmetry, the coefficients $\left(\tilde{a}_{n}, \tilde{b}_{n}\right)$ can be derived by comparing (12) with (13), (18) with (19), (20) with (21) or (22) with (23). One obtains for all cases

$$
\begin{aligned}
& K_{n} b_{n}=R_{n} \hat{b}_{n}, \\
& K_{n} a_{n}=R_{n} \hat{a}_{n} .
\end{aligned}
$$

With (14) it follows that

$$
\begin{aligned}
& \tilde{a}_{n}=\frac{K_{n}}{R_{n}} \cdot \frac{a_{n} T_{n}-b_{n} S_{n}}{S_{n}^{2}+T_{n}^{2}} \\
& \tilde{b}_{n}=\frac{K_{n}}{R_{n}} \cdot \frac{a_{n} S_{n}+b_{n} T_{n}}{S_{n}^{2}+T_{n}^{2}}
\end{aligned}
$$

\section{ACKNOWLEDGMENTS}

We are thankful to the members of the RHIC spin collaboration group for discussions.

\section{REFERENCES}

[1] J. Rossbach and P. Schmüser, "Basic course on accelerator optics", Fifth General Accelerator Course, University of Jyväskylä, Finland, CERN 94-01 (1994).

[2] M. Abramowitz and I. Stegun, "Handbook of Mathematical Functions", Dover, New York (1972). 


\section{DISCLAIMER}

This report was prepared as an account of work sponsored by an agency of the United States Government. Neither the United States Government nor any agency thereof, nor any of their empleyees, makes any warranty, express or implied, or assumes any legal liability or responsibility for the accuracy, completeness, or usefulness of any information, apparatus, product, or process disclosed, or represents that its use would not infringe privately owned rights. Reference herein to any specific commercial product, process, or service by trade name, trademark. manufacturer, or otherwise does not necessarily constitute or imply its endorsement, recommendation, or favoring by the United States Government or any agency thereof. The views and opinions of authors expressed herein do not necessarily state or reflect those of the United States Government or any agency thereof. 